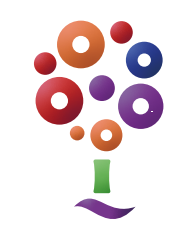

\title{
Luis Sandoval: hombre ilustre de un poblado patrimonio cultural de la humanidad
}

\section{Luis Sandoval: distinguished man from a town that is a World Heritage Site}

\author{
Rosalinda Sandoval Orihuela \\ rosalindasandoval689@gmail.com \\ Universidad Nacional Autónoma de México, \\ México \\ https://orcid.org/0000-0002-0008-051X
}

Sandoval Orihuela, R. (2021). Luis Sandoval: hombre ilustre de un poblado patrimonio cultural de la humanidad. Oralidad-es, 7, 1-9. https://doi.org/10.53534/oralidad-es.v7a2

Fecha de recepción: 25 de julio de 2021 / Fecha de aceptación: 11 de noviembre de 2021

(c) (1) 


\section{Abstract}

This article is the result of talks that were informally held with friends and acquaintances of the character (that is why the permits for publication are not attached), where the most important data of his biography, and therefore of his work, are highlighted; with the aim of showing why he is considered an illustrious man of Xochimilco. We also include information from conversations, which we heard from the 1980s to 2018, and from testimonies that Luis Sandoval expressed in his own voice at the time. This highlights the context in which he was born, grew up, trained as a professional and participated in countless jobs as well as in cultural events in favor of the town where he grew up and died. This period covers from 1930 to 2018.

Keywords

Culture; Xochimilco; talks; Luis Sandoval

\section{Resumen}

El presente artículo es resultado de pláticas que de manera informal (por eso no se adjuntan los permisos para publicación) se realizaron con amigos y conocidos del personaje en donde se destacan los datos más importantes de su biografía, y por ende de su obra; con el objetivo de demostrar porque es considerado un hombre ilustre de Xochimilco. También incluimos información de conversaciones, que escuchamos desde los años 80 hasta el 2018, y de testimonios que en su momento Luis Sandoval expresó de propia voz. Así se destaca el contexto en que nació, creció, se formó como profesionista y participó en innumerables trabajos así como en los eventos culturales en favor del pueblo en donde creció y murió. Dicho periodo abarca de 1930 a 2018.

Palabras Clave

Cultura; Xochimilco; pláticas; Luis Sandoval 


\section{Los primeros pasos}

Luis Sandoval Fraga, nació el 2 de julio de 1930, en Zurumucapio, un pequeño poblado del estado de Michoacán, de los Estados Unidos Mexicanos. Fue el tercero de siete hermanos, hijo de Imelda Fraga y Froylán Sandoval, este último un indígena purépecha que además de hablar su idioma original (purépecha), también dominaba el español.

$\mathrm{Al}$ iniciar la revolución mexicana en 1910, sus padres se unieron a la causa, en donde fueron compañeros de lucha de Lázaro Cárdenas, quien más adelante sería presidente de México (19341940).

Cuando terminó el movimiento armado, en medio de un país convulso y debido a la precaria situación económica de la familia, decidieron trasladarse en 1934 a la capital del país y, es así como llegan el matrimonio de Froylán e Imelda con: Eréndira, Guadalupe, Luis y Gustavo a Xochimilco.

A decir de Luis Sandoval, cuando llegaron a Xochimilco le impactaron muchas cosas: una fuente con forma de un niño que había en el jardín Juárez, ya que él nunca había visto una; que todos se conocieran y se saludaran como en su pueblo.

Que cuando lo mandaban por el pan, la leche y a acarrear agua, todavía por las calles sin pavimentar se encontraba con figuras prehispánicas que recogía para jugar con ellas, sin saber el invaluable valor que tenían en sus manos.

En esta parte cabe destacar que fue cuando conoció, haciendo las mismas labores a José Farías Galindo, quien más tarde por sus estudios recibiría entre otros el título de Cronista de Xochimilco y fuera autor de innumerables libros así como de canciones dedicadas a la Cementera de flores.
Sandoval también nos platicó que estaba encantado viendo la gran cantidad de ojos de agua y ni qué decir de los canales, que en aquellos años eran cristalinos; que en esos canales aprendían a nadar los niños de una manera muy regionalista: desde que empezaban a caminar, eran atados con un rebozo a la parte trasera de una canoa y poco a poco los iban soltando hasta que nadaban solos.

Su peor travesura era irse a los mencionados canales con sus amigos, zambullirse y sacar las almejas que en los años 40 todavía existían a las orillas de las chinampas. Abrirlas, comerlas con sal y limón. Más tarde, al llegar a la casa, recibir la paliza correspondiente ya que estaba prohibido por sus padres acercarse a la zona.

\section{Camino al Internado Madero}

Al aumentar la familia, aumentaron también las carencias y Luis Sandoval junto con sus hermanos mayores y sus hermanas, fueron inscritos en el internado Madero y en el García Conde respectivamente.

El objetivo de estos internados era brindar ayuda a los huérfanos de la Revolución y a los hijos de los veteranos de la misma, lo anterior lo escuchamos directamente de los labios de Luis Sandoval Fraga.

Una anécdota que nos platicó fue que cuando su padre lo llevó al internado, percibía el lugar frío $y$, feo lo cual le provocaba miedo, pero en esa época no se estilaba poner en duda las decisiones de los progenitores, y no tuvo otra opción más que guardar silencio.

Aunque la educación en el lugar era muy estricta, militarizada, se hizo de muchos amigos y, los fines de semana que podían ir los alumnos a sus casa, él ya no deseaba hacerlo, ya que en su centro de estudios contaba con comida segura. 
Allí concluyó la primaria que había iniciado en la escuela Fernando Celada, en Xochimilco.

Los estudios posteriores los realizó en la actual secundaria \#4 Moisés Sáenz ubicada en la colonia Santa María la Ribera.

\section{Su vida personal}

Su carácter bohemio, su afición a la tauromaquia y la charrería hizo que algunas gentes de Xochimilco le llamaran "El 1000 amigos", cabe destacar que siguiendo las tradiciones pueblerinas, siempre traía consigo un paliacate, con el que solía limpiarse la nariz y por ello le apodaron "El Indio”, sobrenombre del cual siempre se sintió orgulloso, ya que el percibía que hacían alusión a su padre, que como ya mencionamos antes era un indígena purépecha.

Un buen bohemio que conoció a otros bohemios; la mayoría en la casa de José Farías Galindo, donde convivió muy estrechamente, en los años 50, con Güicho Cisneros, compositor e intérprete de "Gema" "Tres regalos"... siempre estaba rodeado de camaradas que también fueron destacados cada uno en su área:

- El Profr. Noé Alquicira, excelente maestro y profundo amante de su tierra

- El C. Emilio Arriaga, Jefe de la oficina en Xochimilco del casi extinto sistema de comunicación telégrafos nacionales

- El Profr. José Farías Galindo, entrañable amigo y cronista de Xochimilco

- Los C. Víctor Hernández y Rodolfo Fuentes, ambos campeones mundiales de frontón

- El reconocido Profr. Isaías Morones Escamilla

- El periodista Luis Ramos, director de la revista Rutas

- El C. Gelasio Rioja
En 1965 se casó con una profesora oriunda de Xochimilco, Isaura Orihuela Valverde, procrearon dos hijos: Froylán quien nació el 23 de noviembre de 1965 y Rosalinda quien llegó al mundo el 3 de noviembre de 1968.

Ambos como primera profesión son maestros de primaria y el varón como segunda carrera estudió la licenciatura en Geografía en la Facultad de Filosofía y Letras de la Universidad Nacional Autónoma de México (UNAM).

Rosalinda es licenciada y maestra en Ciencias de la Comunicación por la Universidad Autónoma Metropolitana (UAM) y por la UNAM respectivamente.

\section{Imagen 1.}

Luis Sandoval Fraga a los 77 años.

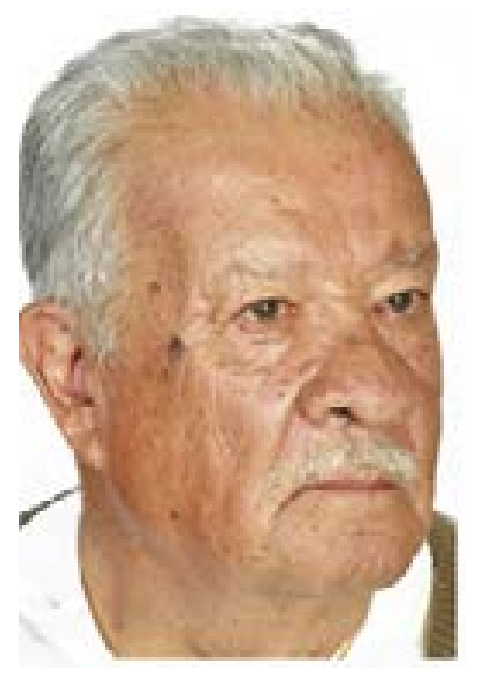

Nota: Sandoval, R. (2007) Archivo personal.

Luis Sandoval siempre nos manifestó su deseo de ser abuelo, pero su única nieta Anam Elisa Sandoval Jiménez, hija de Froylán Sandoval y de la pedagoga Beatriz Jiménez, nació dos años después de su muerte.

Su sepelio se realizó como él lo deseo: con la más absoluta discreción: y hasta el día de hoy todavía recibimos reclamos y pésames por parte de la gente que no se enteró y por lo tanto no pudo despedirlo como hubieran querido: 
Con homenajes, con placas alusivas en los lugares donde trabajó; que lo recuerdan cuando visitan lugares en donde estuvieron con él; que les hubiera gustado decirle "adiós amigo".

Que si pudieran verlo alguna vez, le dirían que hace falta en el pueblo, que lo escucharían con más atención, que platicarían acerca del deterioro que en el que se encuentra Xochimilco

\section{Cementera de flores}

Aunque actualmente hay discrepancias en relación con el significado de Xochimilco, desde que tenemos uso de razón, en los eventos delegacionales, en las escuelas, e incluso en algunos espectaculares se ha dicho que es Cementera de Flores.

\section{Imagen 2.}

Glifo de Xochimilco

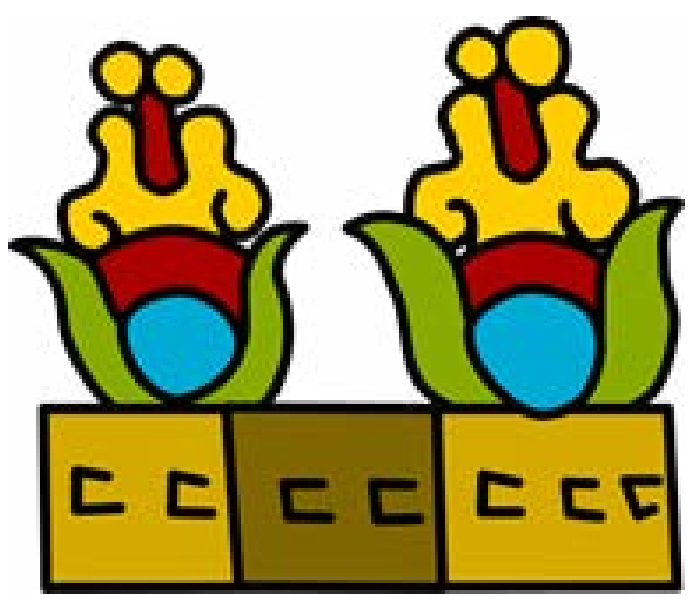

Nota: imagen tomada de https://www.google.com/search?q=glifo+xochimilco\&rlz
Imagen 3.

Explicación del glifo de Xochimilco

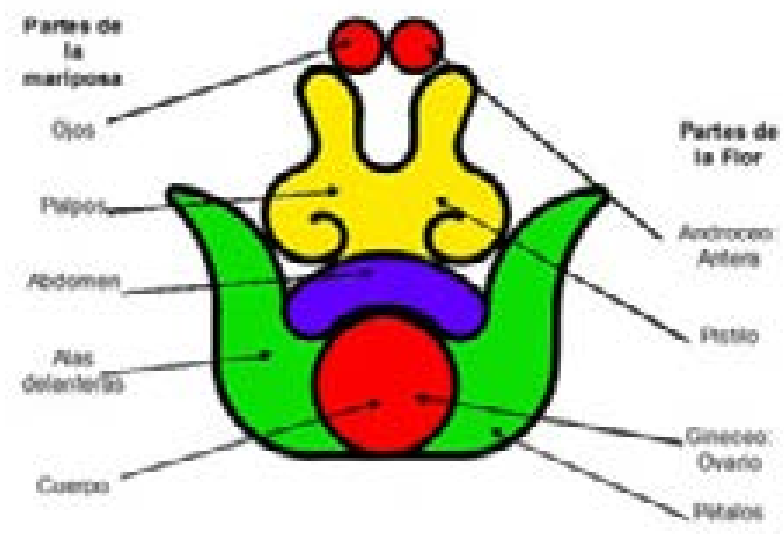

Nota: imagen tomada de https://tonalpohualli260.wordpress.com/2009/11/27/significado-del-simbolo-prehispanico-de-xochimilco

Imagen 4.

Tierra fértil para sembrar

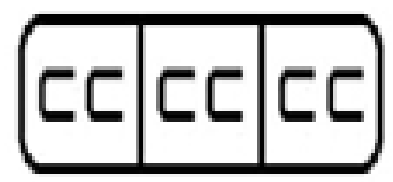

Nota: imagen tomada de https://tonalpohualli260.wordpress.com/2009/11/27/significado-del-simbolo-prehispanico-de-xochimilco 


\section{Xochimilco fue nombrado Patrimonio Cultural de la Humanidad en 1987}

\author{
Imagen 5. \\ Inscripción de Xochimilco como Patrimonio Cultural de la \\ Humanidad
}

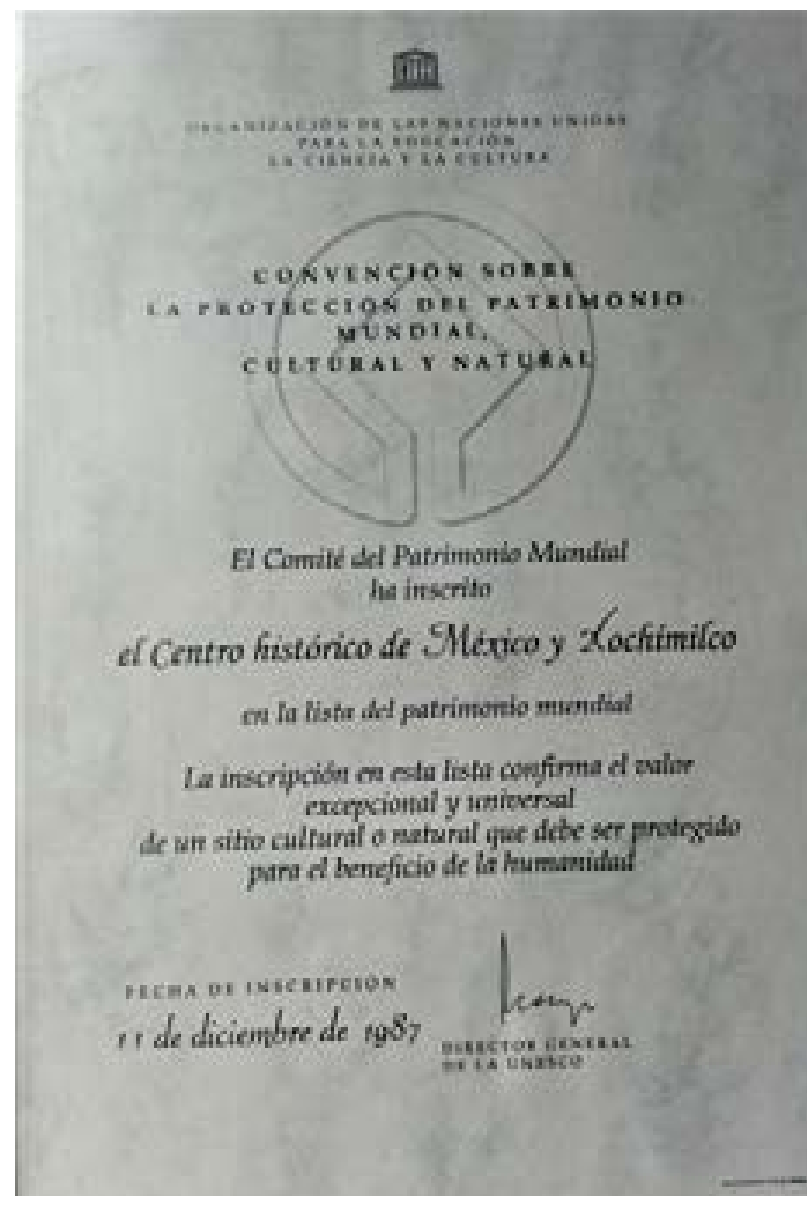

Nota: imagen tomada de Zaldívar, L. (1987) Archivo personal.

Definitivamente, la Ciudad de México no puede concebirse sin Xochimilco, esta alcaldía es un referente turístico, histórico y cultural. Existen Cuahilama, un centro ceremonial de ruinas prehispánicas; un museo arqueológico integrado en su totalidad por el Profr. José Farías Galindo.

Los Xochimilcas fueron los creadores del procedimiento de siembra llamado chinampa, palabra que proviene del náhuatl chinampan, que significa en ia ceica de cañas. Consiste en crear lotes de tierra firme dentro del agua, fueron hechas de manera manual en zonas de poca profundidad. El objetivo era ganar espacio en aquellos años; al abundante líquido. Se construyeron para sembrar plantas, verduras y hortalizas y es por este sistema de cultivo que le valió a Xochimilco ser considerado Patrimonio Cultural de la humanidad.

Los lugareños platican que este sistema se ha intentado implantar en otros países, pero que no ha dado resultado.

Para desgracia de nosotros, los canales que en algún tiempo fueron aguas cristalinas ya son aguas negras; el nivel cada vez desciende más e incluso hay lugares por donde ya se puede pasar caminando.

Los fines de semana por las noches, las trajineras se han vuelto bares flotantes sin ninguna regulación.

\section{La lucha por el estudio y el trabajo}

Continuando con las precariedades, logró recibirse como ingeniero civil, egresado de la Escuela Superior de Ingeniería y Arquitectura del Instituto Politécnico Nacional; y mientras estudiaba, también trabajaba dando clases particulares de: dibujo técnico, matemáticas; mozo de cuadra, además de las obligatorias labores domésticas.

Ya siendo ingeniero realizó muchas obras tanto remuneradas como no remuneradas, en la capital y en el interior del país. Por su experiencia laboral se especializó en caminos, puentes y vialidad

Para 1955 participó en la reparación y ampliación de la carreta México-Acapulco. Puerto que hasta hoy es de los más importantes destinos turísticos del país. 
En Xochimilco dirigió la construcción de La plaza de toros, la arena de lucha, y gratuitamente la cripta "Del poeta del pueblo" Fernando Celada.

Durante 1967 colaboró en la construcción del Centro Deportivo Xochimilco.

En la década de los 80, demostró que las aguas negras de los pobladores de la zona chinampera iban directo a los canales e hizo una propuesta para que estos se rescataran en dos meses. Dicha propuesta no fue tomada en cuenta por las autoridades, ya que no les interesó regularizar el drenaje ni hacer lo que ellos llamaron una costosa inversión.

También presidió y participó grupos para el rescate y conservación de Xochimilco como: juntas de vecinos, apoyo a deportistas, comerciantes...

Promovió junto con otros personajes la construcción de la Rotonda de los personajes ilustres en el centro de Xochimilco.

Desde principios de los años 60 hasta 1986, fue catedrático en la Escuela Superior de Ingeniería y Arquitectura (E.S.I.A.) del Instituto Politécnico Nacional (I.P.N.)

Durante su época de catedrático le tocaron vivir acontecimientos muy fuertes como la matanza estudiantil de 1968 y de 1971, lo que recordaba con gran dolor y nos mencionó que hubo estudiantes que no volvió a ver; tal vez porque estaban muertos, desaparecidos o simplemente decidieron, por su propia seguridad, abandonar la escuela.

Colaboró en la construcción de La Pista Olímpica de Remo y Canotaje Virgilio Uribe, que se ubica en Cuemanco y se inauguró en los Juegos Olímpicos de 1968.

También participó en el patronato Fernando Celada Miranda: que se constituyó como tal en 1972 y en el que trabajaron arduamente los profesores: Isaías Morones, José Farías Galindo, Antonio Escobar Yedra, Félix González Sando- val; el capitán Santos Fernández Cerón; el pintor Rubén Poblano Cordero, el licenciado Luis Sevilla, el periodista Juan Sevilla Medina, poeta José Mendoza y el ingeniero Rafael Sandoval. Y es por esta obra por la que se le recuerda más en Xochimilco.

Estuvo en el Consejo Cultural de Xochimilco, que en los años 76 era el único organismo autónomo para las mejoras físicas de la delegación.

A mediados de la década de los 80, dirigió gratuitamente la construcción del jardín de niños Isaías Morones Escamilla, en un terreno donado por este último, el predio está ubicado en el pueblo de San Andrés Ahuayucan. Cabe destacar que el Profr. Morones primeramente fue su mentor en la educación primaria y partir de 1975 se hicieron compadres.

Fue en la octava década del siglo pasado, cuando de manera altruista ayudó al Profr. José Farías Galindo a integrar el archivo histórico de Xochimilco.

A principios de la misma década, trabajó en la ampliación de la línea 3 de metro, de la estación Zapata a Ciudad Universitaria, desafortunadamente dos infartos consecutivos lo hicieron renunciar a ese cargo.

Después del terremoto que sacudió al entonces Distrito Federal, en 1985, trabajó en los Comités de reparación de vivienda, en la delegación Benito Juárez, una de las más afectadas por el siniestro. El gobierno solicitó el apoyo al Instituto Politécnico Nacional, quien a su vez mandó a sus mejores peritos.

Cuando alguna autoridad en los años 90 quiso arbitrariamente, y para justificar presupuestos; desaparecer una glorieta en donde se encuentra el lote de los personajes ilustres, con argumento de mejorar la circulación de vehículos; el experto en ingeniería vial, apoyado por gente del pueblo, evitó su demolición. 
Imagen 6.

Acreditación de Luis Sandoval Fraga como Apoyo Técnico en Vivienda

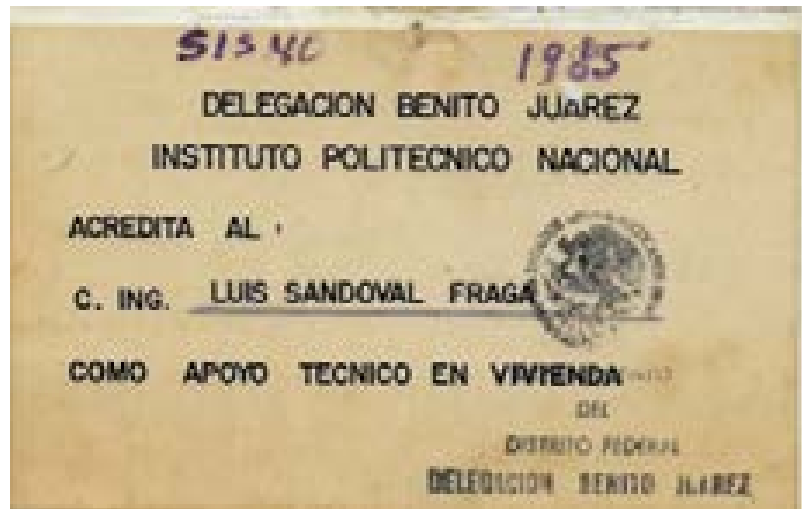

Imagen tomada de: Delegación Benito Juárez. (1985). Instituto Politécnico Nacional. Apoyo Técnico en vivienda. (Credencial).

Anualmente participaba en la organización del festival de La Flor más Bella del Ejido y en la Ofrenda Delegacional del día de Muertos, también aportaba discursos ilustrativos de estas fechas.

Fue Presidente y miembro activo de la Asociación Pro-dignificación del Jubilado del ISSSTE (Instituto de Seguridad y Servicios Sociales de los Trabajadores del Estado)

Cuyo objetivo era igualar los salarios de los jubilados con los trabajadores en activo, ya que así lo marca la ley; sin embargo, esta acción hasta el día de hoy no ha podido cumplirse.

En esta etapa (1990-200), también gestionó credenciales de la Tercera edad para que los adultos mayores no pagaran pasaje en el metro, camiones urbanos, tren ligero; la mitad del costo en los camiones foráneos...

\section{Conclusiones}

- Desafortunadamente las nuevas generaciones poco saben de los hombres y mujeres que han luchado por conservar la riqueza natural y cultural de Xochimilco.

- Ante el panorama tan desolador de contaminación de los canales, altos índices delincuenciales hacen falta más personas que luchen a favor de la Cementera de Flores.

- Luis Sandoval realizó labores tangibles e intangibles en pro de Xochimilco, por lo que se promovió que se escribieran libros al respecto que en los años 50 , cuando empezó su labor época eran muy pocos.

- Con las acciones de hombres como el ingeniero Sandoval, podemos darnos cuenta de que Xochimilco es más que trajineras, paseos para que los jóvenes fuereños se emborrachen y se ahoguen.

- El ingeniero Sandoval tuvo la visión y la agudeza para planificar y llevar a cabo acciones en favor de los Xochimilcas. 


\section{Referencias}

Fototeca

Sandoval, R. (2007) Archivo personal.

Zaldívar, L. (1987) Archivo personal.

\section{Páginas Web}

https://www.google.com/search?q=glifo+xochi$\underline{\text { milco\&rlz }}$

https://tonalpohualli260.wordpress. com/2009/11/27/significado-del-simbolo-prehispanico-de-xochimilco

\section{Otras fuentes}

Delegación Benito Juárez. (1985). Instituto Politécnico Nacional. Apoyo Técnico en vivienda. (Credencial). 